\title{
Influence of Xingnaojing Injection on the Expression of c-fos and c-jun Proteins in Brains of Rats in a Kindling Model of Epilepsy Chronically Induced by Pentetrazol
}

\author{
Ji-Wei Cheng Yu Bai Xiao-Jing Zhang Li-Jun Zhang Yu-Qing Hou \\ Department of Neurology, Putuo Hospital, Shanghai University of Traditional Chinese \\ Medicine, Shanghai, China
}

Key Words

Epilepsy $\cdot$ Kindling model $\cdot$ Xingnaojing injection $\cdot c$-fos $\cdot c$-jun

\begin{abstract}
Objective: To investigate the molecular biological mechanism of the antiepileptic effect of Xingnaojing (XNJ) injection on a rat kindling model of epilepsy (KME). Methods: Fifty healthy 6-week-old male Sprague-Dawley rats were divided into the following five groups: blank control (BC) group, model (M) group, XNJ injection (XI) group, phenobarbital injection (PI) group and XNJ combined with phenobarbital (XP) group. There were 10 rats in each group. The intervention drugs were administered $30 \mathrm{~min}$ before the model-building drugs once a day for 5 weeks. The model-building drug pentetrazol was given to each group as an intraperitoneal injection $30 \mathrm{~min}$ after the use of the intervention drug once a day for 4 weeks for KME establishment (except for the BC group). The BC group was given physiological saline instead. All drugs were injected intraperitoneally. The behaviors of each group of rats were observed after the use of the model-building drugs for $1 \mathrm{~h}$ every day. The last kindling test was carried out at the end of week 5. Then, c-fos and c-jun protein expressions in the rat brains of each group were observed and analyzed by immunohistochemistry at the end of the experiment. Results: There was a large number of cells positive for the c-fos and c-jun proteins in the rat brains of the M group. Compared with the M group, the expression level of the c-fos and cjun proteins was lower in the rat brains of the XI and PI groups $(p<0.01)$. There was no statistical difference between the XI and PI groups ( $p>0.05$ ). The number of positive cells in the rat brains of the XP group was even smaller than that of the XI or PI groups. Conclusion: The antiepileptic effect of the XNJ injection on the rat KME is probably related to its interruption function on the expression of the c-fos and c-jun proteins in rat brains.
\end{abstract}




\section{Introduction}

Epilepsy is the second most common neurological disease in the world. There are about 50 million patients with epilepsy and about half of them live in Asia [1]. Western antiepileptic drugs can control most epilepsy. However, there are still about $20 \%$ of epilepsy cases that cannot be well controlled. In recent years, side effects of Western antiepileptic drugs, such as liver damage and cognitive impairment, have drawn our attention. It is necessary to find new antiepileptic drugs with higher curative benefits and less side effects. Traditional Chinese medicine can improve various symptoms, particularly in neurological diseases. Our previous study [2] has shown that Xingnaojing (XNJ) injection can inhibit the formation of epileptogenic focus and epileptic seizure. However, the mechanism is unknown. In the last 10 years, attention has been paid to the relationship between abnormal gene expression and epilepsy. Several studies [3-5] showed that there is a close relationship between immediate early genes (IEGs, c-fos and c-jun, etc.) and seizures. Seizures can cause increased expression of c-fos and c-jun. Thus, long-time expression of c-fos and c-jun might lead to the formation of an epileptogenic focus and epileptic seizure. In this study, we observed the effect of XNJ injection on the expression of c-fos and c-jun proteins in the brain of rats with epilepsy induced by pentetrazol (PTZ) and explore the possible antiepileptic mechanism of XNJ injection.

\section{Materials and Methods}

Animals

Fifty healthy 6-week-old male Sprague-Dawley rats (clean level, mean weight $\pm S D=180 \pm 20 \mathrm{~g}$ ) were supplied by Shanghai Super-B\&K Laboratory Animal Co. Ltd. [license No: SCXK (Hu) 2003-000].

Drugs and Reagents

The following drugs and reagents were used: XNJ injection (Yunnan Dali Pharmaceutical Co. Ltd., batch No.: 070225), PTZ (Sigma Company, batch No.: 070101), phenobarbital sodium injection (Shanghai Xinya Pharmaceutical Co. Ltd., batch No.: 061104), rabbit anti-rat c-fos polyclonal antibody (Shanghai Bogu Biological Technology Co. Ltd., batch No.: SA07012532), rabbit anti-rat c-jun polyclonal antibody (Shanghai Bogu Biological Technology Co. Ltd., batch No.: SA06120153), and anti-antibody kit (Fuzhou Maixin Biological Technology Co. Ltd.).

\section{Equipment}

The following equipment was used: microtome (version RM2235, Germany), optical microscope (OLYMPUS, version BX51TF, Japan), and HMIAS-2000 high-definition color medical image analysis system (Wuhan Qianping Imaging Technology Co. Ltd.).

Groups

Fifty healthy male Sprague-Dawley rats were randomly divided into the following five groups: blank control (BC) group, model (M) group, XNJ injection (XI) group, phenobarbital injection (PI) group and XNJ combined with phenobarbital (XP) group. There were 10 rats in each group.

Drug Intervention and Model Establishment

Drug Intervention. The intervention drugs were administered $30 \mathrm{~min}$ before the model-building drugs once a day. The $\mathrm{BC}$ and $\mathrm{M}$ groups were given physiological saline $\left(15 \mathrm{ml} \cdot \mathrm{kg}^{-1} \cdot \mathrm{day}^{-1}\right)$. The XI group received XNJ injections $\left(15 \mathrm{ml} \cdot \mathrm{kg}^{-1} \cdot \mathrm{day}^{-1}\right)$. The PI group was given phenobarbital injections $\left(30 \mathrm{mg} \cdot \mathrm{kg}^{-1} \cdot \mathrm{day}^{-1}\right)$. The XP group was administered both XNJ and phenobarbital injections according to the doses of the XI and PI groups. All drugs were injected intraperitoneally. The intervention drugs were given for 5 weeks.

Model Establishment. PTZ was given to each group $\left(35 \mathrm{mg} \cdot \mathrm{kg}^{-1} \cdot \mathrm{day}^{-1}, 1 \%\right)$ through intraperitoneal injection $30 \mathrm{~min}$ after the use of the intervention drug once a day for the rat kindling model of epilepsy (KME) 


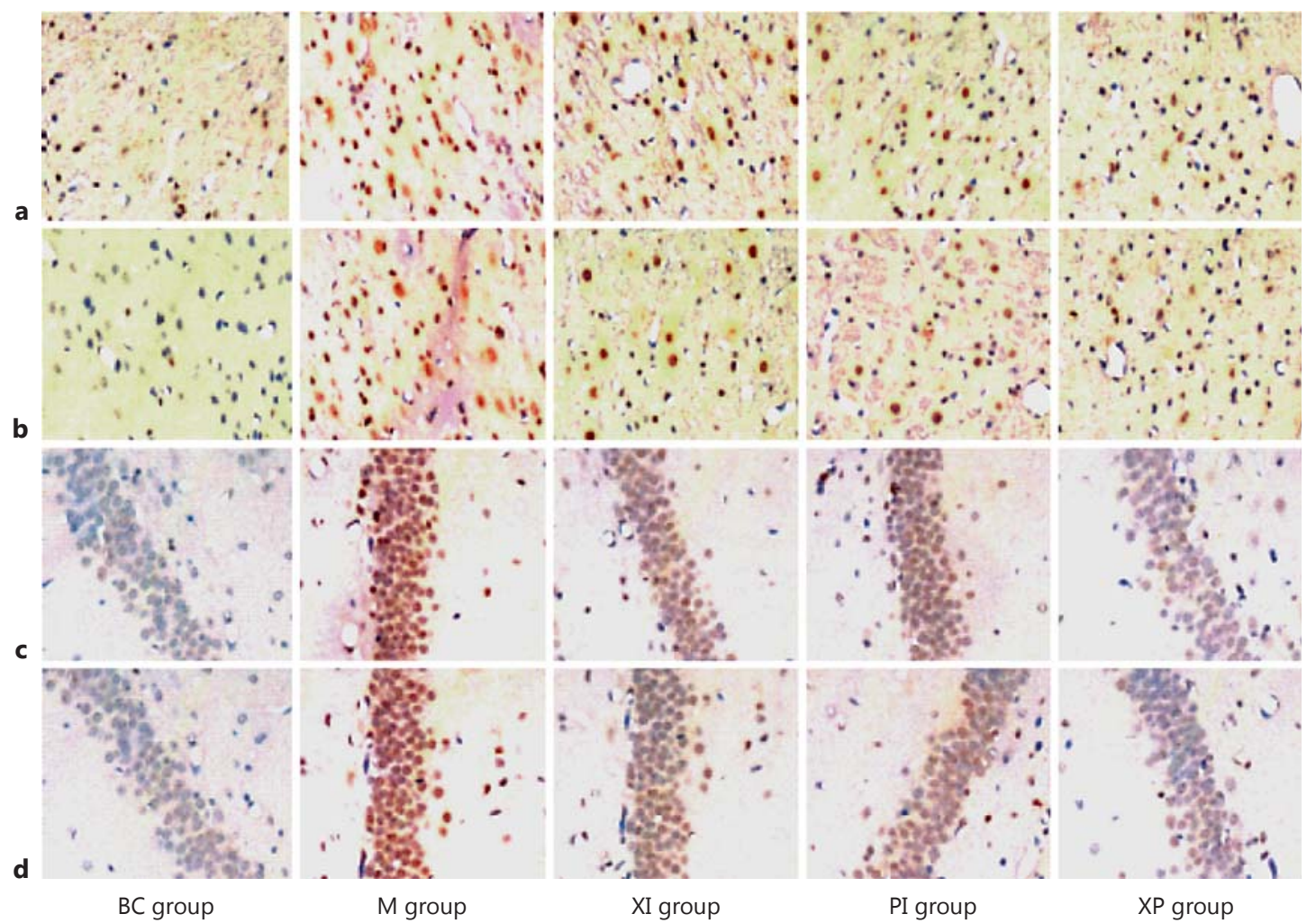

Fig. 1. Expression of c-fos and c-jun proteins in the cortex and hippocampus. a c-fos protein in the cortex. b c-jun protein in the cortex. c c-fos protein in the hippocampus. d c-jun protein in the hippocampus. BC group = blank control group; $\mathrm{M}$ group = model group; XI group = Xingnaojing injection group; PI group = phenobarbital injection group; XP group $=\mathrm{XNJ}$ injection combined with the phenobarbital group. Immunohistochemisty $\times 200$.

establishment (except for the BC group). The BC group was given physiological saline $\left(3.5 \mathrm{ml} \cdot \mathrm{kg}^{-1} \cdot \mathrm{day}^{-1}\right)$ instead. Then, the behavior of each rat was observed and recorded for $1 \mathrm{~h}$ every day according to the Smialowski-6-rank method. The model-building drugs were used for 4 weeks and then stopped for 1 week. The last kindling test was carried out at the end of week 5. The animals that displayed level 2 and above seizures for 5 times in a row were recognized as the succeed models [6].

\section{c-fos and c-jun Protein Determination}

The rats were sacrificed and brain tissue was collected after the last kindling test and EEG data acquisition. The specific method was as follows: first, the rats were anesthetized by napental ( $40 \mathrm{mg} / \mathrm{kg})$. Then, $200 \mathrm{ml}$ physiological saline was rapidly infused into the rats through the abdominal aorta until supernatant liquid flowed out. Afterwards, about $300 \mathrm{ml}$ paraformaldehyde (4\%) was slowly infused into the rats until they became stiff. Then, the heads of the rats were sheared and the brains were taken out. The brains were put into sucrose $(200 \mathrm{~g} / \mathrm{l})$ at $4{ }^{\circ} \mathrm{C}$ for one night. The following day, the brains were cut into coronal slices. The thickness of the slices was $2 \mathrm{~mm}$. The slices included the cerebral cortex and the hippocampus. The specimens were evaporated in graded alcohol and then embedded in paraffin. Finally, the paraffin blocks with the specimens were consecutively cut into coronal slices. The thickness of the slices was $4 \mu \mathrm{m}$. Then, the cells positive for c-fos and c-jun were measured using the streptavidin-peroxidase two-step method. The negative nucleuses are blue or light blue and the positive nucleuses are yellow or brown. The positive nucleuses were counted with HMIAS-2000 the high-definition color medical image analysis system. Five equal areas were randomly selected from the corresponding parts of each section of the cerebral cortex and hippocampus to count the number of positive cells. In addition, the mean number of positive cells from the five areas was recognized as the number of positive cells of the rat. 
International

Table 1. Results of the expression of c-fos protein in rat brains

\begin{tabular}{l|l}
\hline DOI: $10.1159 / 000362637$ & $\begin{array}{l}\text { C 2014 S. Karger AG, Basel } \\
\text { www.karger.com/imi }\end{array}$ \\
\hline Cheng et al.: Influence of XNJ Injection on the Expression of c-fos and c-jun Proteins in
\end{tabular}

Brains of Rats in a KME Chronically Induced by PTZ

\begin{tabular}{lrcc}
\hline Groups & $\mathrm{n}$ & \multicolumn{2}{l}{ Positive cells, $\mathrm{n}$} \\
\cline { 3 - 4 } & & cerebral cortex & hippocampus \\
\hline BC & 10 & $18.72 \pm 11.87$ & $15.88 \pm 12.79$ \\
M & 9 & $112.27 \pm 18.55^{\mathrm{a}}$ & $104.33 \pm 19.76^{\mathrm{a}}$ \\
XI & 10 & $60.86 \pm 21.42^{\mathrm{b}, \mathrm{c}}$ & $56.08 \pm 22.30^{\mathrm{b}, \mathrm{c}}$ \\
PI & 10 & $51.88 \pm 19.48^{\mathrm{b}, \mathrm{c}}$ & $49.52 \pm 20.54^{\mathrm{b}, \mathrm{c}}$ \\
XP & 10 & $26.82 \pm 13.67^{\mathrm{b}}$ & $22.86 \pm 14.92^{\mathrm{b}}$ \\
\hline
\end{tabular}

Values are means \pm SD.

${ }^{\mathrm{a}} \mathrm{p}<0.01$, compared with the BC group. ${ }^{\mathrm{b}} \mathrm{p}<0.01$, compared with the $\mathrm{M}$ group. ${ }^{\mathrm{c}} \mathrm{p}<0.01$, compared with the XP group.
Table 2. Results of the expression of c-jun protein in rat brains

\begin{tabular}{lrcc}
\hline Groups & $\mathrm{n}$ & Positive cells, $\mathrm{n}$ & \\
\cline { 3 - 4 } & & cerebral cortex & hippocampus \\
\hline BC & 10 & $17.16 \pm 13.16$ & $14.96 \pm 12.00$ \\
M & 9 & $109.20 \pm 19.34^{\mathrm{a}}$ & $103.38 \pm 19.80^{\mathrm{a}}$ \\
XI & 10 & $56.94 \pm 21.91^{\mathrm{b}, \mathrm{c}}$ & $55.22 \pm 22.43^{\mathrm{b}, \mathrm{c}}$ \\
PI & 10 & $50.64 \pm 19.78^{\mathrm{b}, \mathrm{c}}$ & $47.78 \pm 20.66^{\mathrm{b}, \mathrm{c}}$ \\
XP & 10 & $24.26 \pm 14.70^{\mathrm{b}}$ & $22.66 \pm 14.12^{\mathrm{b}}$ \\
\hline
\end{tabular}

Values are means \pm SD.

${ }^{\mathrm{a}} \mathrm{p}<0.01$, compared with the BC group. ${ }^{\mathrm{b}} \mathrm{p}<0.01$, compared with the $\mathrm{M}$ group. ${ }^{\mathrm{c}} \mathrm{p}<0.01$, compared with the XP group.

\section{Statistics}

SPSS14.0 was used to determine the difference. The experimental data were expressed as means \pm SD. Analysis of variance was used to compare the number of cells positive for the c-fos and c-jun proteins among the five groups, and the least significant difference was used for comparison between each group. The Spearman method was used to analyze the correlation between the number of positive cells in the rat brain and the level of epileptic seizures. The comparison of the number of cells positive for the c-fos and c-jun proteins was our main index. Significant differences was set at a p value of $<0.05$.

\section{Results}

\section{Animals}

There were 50 rats in the experiment, and 49 rats were included in the result analysis except for 1 rat of the $M$ group which died because of epilepsia gravior.

\section{c-fos Protein Expressions}

The number of c-fos-positive cells in the rat brains of the $M$ group was higher than that in the $\mathrm{BC}$ group $(\mathrm{p}<0.01)$, and the number of cells positive for the c-fos protein in the rat brains of the XI, PI and XP groups was lower than that in the M group ( $<<0.01)$. There was no statistical difference between the XI and PI groups ( $p>0.05)$. However, the number of $c$-fos-protein positve cells in the rat brains of the XP group was lower than that in the XI and PI groups ( $p<0.01$; table 1; fig. 1 ). 
Cheng et al.: Influence of XNJ Injection on the Expression of c-fos and c-jun Proteins in Brains of Rats in a KME Chronically Induced by PTZ

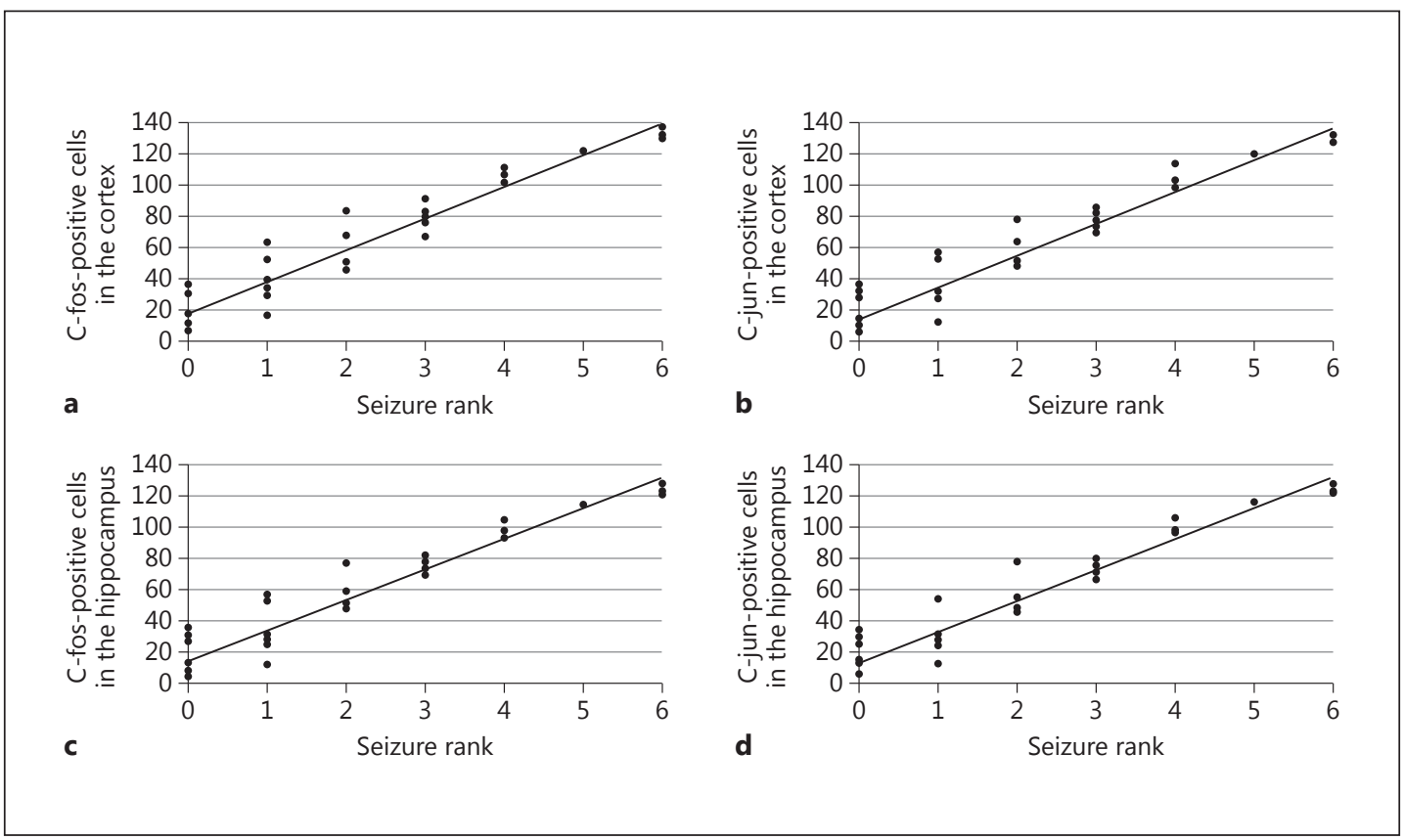

Fig. 2. Correlation between seizure rank and the expression of c-fos and c-jun proteins. a Seizure rank and c-fos-positive cells in the cortex. b Seizure rank and c-jun-positive cells in the cortex. c Seizure rank and cfos-positive cells in the hippocampus. $\mathbf{d}$ Seizure rank and c-jun-positive cells in the hippocampus.

c-jun Protein Expressions

The number of cells positive for the $\mathrm{c}$-jun protein in the rat brains of the $\mathrm{M}$ group was higher than in the BC group ( $<<0.01)$, and the number of cells positive for the $c$-jun protein in the rat brains of the XI, PI and XP groups was lower than that in the M group $(p<0.01)$. There was no statistical difference between the XI and PI groups $(p>0.05)$. However, the number of cells positive for the c-jun protein in the rat brains of the XP group was lower than that in the XI and PI groups ( $\mathrm{p}<0.01$; table 2; fig. 1$)$.

\section{Behaviors}

Five days after the PTZ injection, the first epileptic attack occurred in the M group, and it worsened with more injections. In the M group, rank 6 epileptic attacks were observed 4 weeks later. The times and rank of seizures of the XI and PI groups reduced obviously. The highest level of epileptic attacks was rank 4 which was recorded in the XI and PI groups. The times and rank of seizures of the XP group reduced even more. The highest seizure level of the XP group was rank 3 only.

\section{Correlation between Seizure Rank and the Expression of c-fos and c-jun Proteins}

We performed a Spearman correlation analysis of the expression of c-fos and c-jun proteins in the cerebral cortex and hippocampus and recorded seizure ranks at the end of the experiment. The correlation equation and coefficient of rank correlation were as follows: $\mathrm{y}_{1}=20.18 \mathrm{x}_{1}+17.505, \mathrm{r}_{\mathrm{s} 1}=0.918$ (fig. 2a) $\mathrm{y}_{2}=20.107 \mathrm{x}_{2}+15.175, \mathrm{r}_{\mathrm{s} 2}=0.903$ (fig. 2b); $\mathrm{y}_{3}=$ $19.554 x_{3}+13.503, r_{s 3}=0.911$ (fig. $2 c$ ) $; y_{4}=19.541 x_{4}+13.389, r_{s 4}=0.912$ (fig. $2 d$ ). Therefore, we could confirm that there is a strong and direct correlation between the rank of epileptic attacks and the expression of c-fos and c-jun proteins (fig. 2). 
Cheng et al.: Influence of XNJ Injection on the Expression of c-fos and c-jun Proteins in Brains of Rats in a KME Chronically Induced by PTZ

\section{Discussion}

The animal model is an important approach to the study of a drug effect and its mechanism. The epilepsy model includes the acute convulsion model and the chronic kindling model. The acute convulsion model easily leads to death, and there are some differences between acute convulsion and epilepsy. PTZ is a type of central stimulant with little adverse effect. One study [7] showed that long-term repeated subthreshold doses of stimulus can lead to a prolonged epileptic seizure. Eventually, epileptic seizures turn to epilepsy. This process is called chronic kindling. Once the kindling model is established, its excitability can persist lifelong and attack under certain conditions. It has a high similarity with human epilepsy. At present, the kindling model is considered to be an ideal model of epilepsy. Therefore, this experiment used the rat KME chronically induced by PTZ as experimental object.

One study [8] showed that c-fos and c-jun widely exist in the central nervous system. Under normal circumstances, the level of expression of c-fos and c-jun proteins in the central nervous system is low. However, when the body is stimulated by trauma and chemical use, the expression of c-fos and c-jun proteins will obviously change $[9,10]$. The expression of c-fos and c-jun proteins will show great changes when the body is stimulated by epileptogenic stimulation. When the nervous system is stimulated by some signal, the first-class neuron induces the secretion of neurotransmitters. Neurotransmitters as the first messengers will act on the target cell membrane. Signals are carried into the cell through the cell membrane and activate the second messenger such as $\mathrm{Ca}^{2+}$. The second messenger will be combined with the specific base pair of IEG through different ways, and then it will activate the transcription of IEG. The mRNA produced by the transcription of IEG enters into the cytolymph and produces phosphoproteins such as c-fos and c-jun. The phosphoproteins reenter the nuclear phosphoproteins and become the activated protein-1 of the homodimer or heterodimer through the leucine zipper. As the third messenger, the activated protein- 1 will be combined with the binding site of the late-response gene to regulate the long-time expression of the target gene, which can encode various cellular proteins such as neurotransmitters, neurotrophic factor, neural regulation factors, receptors and synaptic structure protein. Then, the inherent component of neuron and the excitability of neuronal network will change, which will lead to the formation of epileptogenic focus and epileptic seizure. This means that seizures can cause a high expression of c-fos and c-jun, and large and long-time expression of c-fos and c-jun will lead to the formation of epileptogenic focus and epileptic seizures. Thus, the inhibition of seizures can reduce or block the expression of c-fos and c-jun, and decreased c-fos and c-jun expression can prevent the onset of the formation of the epileptogenic focus and epileptic seizure.

The XNJ injection is an improved traditional Chinese medicine preparation of the angong niuhuang pill. It is a traditional Chinese medicine injection which is extracted from musk, gardenia, turmeric, borneol and other precious Chinese herbal medicines. It has the effect of brain-activating, activating blood and resolving stasis, heat-clearing and detoxicating, etc. It is widely used in stroke, drunkenness, coma, convulsion, etc. Basal studies [11] showed that XNJ injection (10-20 $\mathrm{ml} \cdot \mathrm{kg}^{-1} \cdot$ day $^{-1}$ ) has a strong inhibitory effect on the central nervous system, and it can reduce the number of free activities of mice and antagonize mice convulsion induced by strychnine and inhibit electric convulsion of mice. Our previous study found that XNJ injection can delay the appearance of an epileptic attack, reduce the rank of the epileptic attack, prolong its incubation period, shorten its duration and depress the seizure wave amplitude and frequency. It displays a good inhibiting effect in the formation of KME induced by PTZ. The combination of XNJ injection and phenobarbital injection displays an even stronger inhibiting effect in the formation of the KME induced by PTZ. Moreover, we found that there was a strong direct correlation between the rank of epileptic attacks and the expression of c-fos and c-jun proteins. 
Cheng et al.: Influence of XNJ Injection on the Expression of c-fos and c-jun Proteins in Brains of Rats in a KME Chronically Induced by PTZ

\section{Conclusion}

This study demonstrates that c-fos and c-jun proteins are involved in the formation of epileptogenic focus and epileptic seizures. XNJ injection might inhibit the expression of c-fos and c-jun proteins and the formation of epileptogenic focus and epileptic attacks. Combined with the previous results, we can infer that the antiepileptic effect of XNJ may be related to the effect of its blocking the expression of c-fos and c-jun proteins in the brain. The exact mechanism needs further research.

\section{Acknowledgement}

This study has been reviewed by Prof. Weidong Pan (Shanghai, China) and Prof. Jiangang Shen (Hong Kong).

\section{References}

$>1$ Mac TL, Tran DS, Quet F, et al: Epidemiology, aetiology, and clinical management of epilepsy in Asia: a systematic review. Lancet Neurol 2007;6:533-543.

2 Jiwei C, Yu B, Xiaojing Z, et al: Influence of Xingnaojing on the behavior and EEG of the rat kindling model of epilepsy induced by PTZ. J Epileptol Electroneurophysiol 2009;18:67-71.

-3 Zechun P, Houser CR: Temporal patterns of fos expression in the dentate gyrus after spontaneous seizures in a mouse model of temporal lobe epilepsy. J Neurosci 2005;25:7210-7220.

-4 Suzuki J: Investigations of epilepsy with a mutant animal (EL mouse) model. Epilepsia 2004;45:2-5.

5 Hsieh C-L, Lin JJ, Chiang SY, et al: Gastrodia elata modulated activator protein 1 via c-Jun N-terminal kinase signaling pathway in kainic acid-induced epilepsy in rats. J Ethnopharmacology 2007;109:241-247.

6 Li W, Ono J, Walson PD: Establishment of rat PTZ kindling model. Acta Pharmaceutica Sinica 1993;28:487.

7 Dhir A, Naidu PS, Kulkarni SK: Effect of naproxen, a non-selective cyclo-oxygenase inhibitor, on pentylenetetrazol-induced kindling in mice. Clin Exp Pharmacol Physiol 2005;32:574-584.

8 Mihaly A, Szakacs R, Bohata C, et al: Time-dependent distribution and neuronal localization of c-fos protein in the rat hippocampus following 4-aminopyridine seizures. Epilepsy Res 2001;44:97-108.

-9 Werme M, Ringholm A, Olson L, et al: Differential patterns of reduction of NGF1-B, Nor1 and c-fos mRNAs in striatal subregions by haloperidol and clozapine. Brain Res 2000;863:112-119.

10 Silveira D, Sogawa C, et al: The expression of fos following kainic acid-induced seizures is age-dependent. Eur J Neurosci 2002;15:329-344.

11 Mingsan M: Effect of Xingnaojing injection on the central nervous system. Pharmacol Clin Chinese Materia Medica 2004;20:42. 